\title{
Cepa antártica de Bacillus sp., con actividad extracelular de tipo agarolítica y alginatoliasa
}

\section{Antarctic strain of Bacillus sp. with extracellular agarolitic and alginate-lyase activities}

\author{
Paris Lavin ${ }^{1 *}$, Jorge Gallardo-Cerda ${ }^{1}$, Cristian Torres-Diaz ${ }^{2}$, Geraldine Asencio ${ }^{1}$ \& Marcelo \\ GonZALEZ ${ }^{1}$
}

'Laboratorio de Biorrecursos Antárticos, Departamento Científico (DECIEN), Instituto Antártico Chileno, Punta Arenas, Chile. 'Laboratorio de Genómica y Biodiversidad (LGB), Departamento de Ciencias Básicas, Facultad de Ciencias, Universidad del Bío-Bío, Chillán, Chile.

*E-mail: plavin@inach.cl

\begin{abstract}
RESUMEN
Diversas bacterias asociadas a macroalgas pueden usar ficocoloides como fuente de carbono. Las bacterias de la Antártica, poseen características fisiológicas adaptativas que habrían evolucionado para permitir su supervivencia y funcionamiento dentro de las condiciones adversas de ese ecosistema. Por lo tanto, bacterias aisladas desde algas antárticas podrían tener la capacidad de degradar enzimáticamente azúcares complejos a temperaturas inferiores que aquellas aisladas desde zonas más cálidas, lo que potencialmente podría tener aplicaciones en la mejora en procesos industriales que utilizan enzimas. El análisis del gen ribosomal 16S indica que la cepa bacteriana aislada desde algas varadas en la Isla Rey Jorge (Antártica), pertenece al género Bacillus. El sobrenadante libre de células presentó actividad agarasa y alginatoliasa. Se encontraron diferencias significativas en la temperatura óptima para hidrolizar agarosa y alginato, evaluada dentro de un rango de 4 a 30 ${ }^{\circ} \mathrm{C}$. La actividad agarolítica fue mayor a $4{ }^{\circ} \mathrm{C}$, mientras que la actividad alginatoliasa fue mayor a $30{ }^{\circ} \mathrm{C}$. Estos resultados poseen un alto valor biotecnológico y podría ser utilizada con fines industriales.
\end{abstract}

Palabras clave: Bacillus, Antártica, Agarasa, Alginatoliasa.

\begin{abstract}
Several bacteria associated to macroalgae can use phycocolloids as carbon source. Antarctic Bacteria's have physiological characteristics that might have evolved to allow the survival and functioning under the harsh conditions of that ecosystem. Therefore, Antarctic bacteria isolated from algae should have the ability to degrade complex sugars at lower temperatures than those isolated from warmer areas, which may have applications in the improvement of industrial processes that uses enzymes. The bacterial strain isolated from wrack algae, in King George Island, Antarctica, was identified as Bacillus on the base of $16 \mathrm{~S}$ ribosomal gene analysis. The cell-free supernatant of the culture medium showed alginate-lyase and agarase activities. Significant differences in the optimal temperature to hydrolyze agarose and alginate were found within a range of 4 to $30^{\circ} \mathrm{C}$. While the agarase activity was higher at $4^{\circ} \mathrm{C}$, the alginate-lyase activity was higher at $30{ }^{\circ} \mathrm{C}$. Our results have biotechnological value and could be used with industrial aims.
\end{abstract}

KeYwords: Bacillus, Antarctic, Agarase, Alginate-lyase.

\section{INTRODUCCIÓN}

Por más de una década los microorganismos marinos han sido intensamente estudiados debido a que son una importante fuente de nuevos compuestos y enzimas, presentando una gran diversidad estructural, relativamente alta estabilidad y mayores activadades que las enzimas encontradas en plantas o animales (Bull et al., 2000; Donia \& Hamann, 2003; Kin, 2006; Stach, 2010). Entre las enzimas marinas más estudiadas destacan las proteasas, amidasas, lipasas y enzimas de degradación de polisacáridos (quitinasas, alginatoliasas, agarasas, carragenasas e hidrolasas de celulosa) (Zhang \& Kim, 2010), las cuales también han sido sujeto de esfuerzos para la optimización de su actividad través de la ingeniería de proteínas (Sarkar et al., 2010). Un grupo de enzimas con potencial uso biotecnológico son aquellas capaces de digerir ficocoloides, tales como agar y alginatos (azúcares estructurales de algas), las que podrían ser utilizadas para producir oligosacáridos con actividad biológica de aplicación en el área de salud y agricultura. 
Diversos autores han logrado caracterizar diversas enzimas provenientes de géneros bacterianos aislados desde ambientes mesófilos como Alteromonas, Bacillus, Cytophaga, Pseudomonas, Pseudoalteromonas, Streptomyces, Thalasomonas y Vibrio (Aoki et al., 1990; Leon et al., 1992; Parro \& Mellado, 1994; Hosoda et al., 2003; Fu \& Kim, 2010; Li et al., 2011; Kam et al., 2011), la mayoría de las cuales, presentan actividades máximas a temperaturas en torno a los $40-50{ }^{\circ} \mathrm{C}$.

Los procesos industriales en los que se utilizan reacciones mediadas por enzimas se caracterizan por ser energética y económicamente costosos. Consecuentemente, la obtención de enzimas que presenten actividad hidrolítica a baja temperatura podría permitir un ahorro económico y un aumento en la eficiencia para obtener los productos finales (Georlette et al., 2004).

El continente antártico se caracteriza por su larga historia de aislamiento y por las codiciones climáticas extremas -tales como bajas temperaturas, altas temperaturas y baja disponibilidad de agua- que en ella imperan. Estas severas condiciones climáticas pueden haber ejercido fuertes presiones selectivas que pueden haber favorecido la evolución de bacterias con rutas metabólicas únicas capaces de producir enzimas y compuestos de interés biotecnológico (Nichols et al., 2002).

Dado que diversas bacterias asociadas a macroalgas pueden usar los ficocoloides como fuente de carbono (Michel et al., 2006), postulamos que las moléculas exudadas por bacterias aisladas desde algas antárticas tendrían la capacidad de degradar azúcares complejos a temperaturas inferiores a $10^{\circ} \mathrm{C}$.

En este trabajo evaluamos la capacidad del sobrenadante de cultivo de una cepa de Bacillus sp., aislada desde algas en descomposición en la Isla Rey Jorge, (Islas Shetland del Sur, Antártica), para hidrolizar ficocoloides a baja temperatura.

\section{METODOLOGÍA}

Material de ESTUdio

La cepa bacteriana fue aislada desde algas varadas en la Isla Rey Jorge (Figura 1) utilizando un medio de cultivo mínimo R2A sólido, cuya principal fuente de carbono es el agar.

SECUENCIACIÓN DEL GEN RIBOSOMAL 16S Y ASIGNACIÓN TAXONÓMICA

Para la identificación de la bacteria aislada desde algas varadas se amplificó el gen $16 \mathrm{~S}$ mediante el protocolo de PCR recomendado por el fabricante de la enzima Taq DNA polimerasa Kappa (BIOSYSTEM). Este protocolo consistió de una reación que contenia: $14,94 \mu \mathrm{l}$ de $\mathrm{H}_{2} \mathrm{O}$ ultra pura, 2 $\mu \mathrm{l}$ de Taq buffer (10X), 0,4 $\mu \mathrm{l}$ dNTP's, $0,8 \mu \mathrm{l}$ del partidor Forward (27F), 0,8 $\mu \mathrm{l}$ del partidor Reverse (1429R), $0,08 \mu \mathrm{l}$ Taq y $1 \mu \mathrm{l}$ de ADN ( $50 \mathrm{ng} / \mu \mathrm{l}$ ), en un volumen final de total de $20 \mu \mathrm{l}$. El programa de PCR se realizó con el siguiente perfil térmico: 1 ciclo de 5 min a $95^{\circ} \mathrm{C}$, seguido por 28 ciclos de: $95{ }^{\circ} \mathrm{C}$ por $1 \mathrm{~min}, 60^{\circ} \mathrm{C}$ por $30 \mathrm{seg}$ y $72{ }^{\circ} \mathrm{C}$ por $2 \mathrm{~min}$, seguido por un ciclo de extensión final a $72{ }^{\circ} \mathrm{C}$ por $10 \mathrm{~min}$. El producto de PCR $(\sim 1.5 \mathrm{~kb})$ fue visualizado bajo luz UV después de la electroforesis en gel de agarosa al $2 \%$ y teñido con $1 X$ SYBR SafeTM (Invitrogen). La banda obtenida fue purificada con el kit PCR Cleanup (Axygen) y enviada a Macrogen (Corea) para su secuenciación. La asignación taxonómica fue realizada mediante la herramienta de libre acceso BLAST (Basic Local Alignment Search Tool) disponible en el sitio web http://blast.ncbi.nlm.nih.gov/Blast. cgi. El análisis filogenético se realizó utilizando el software Bosque (Ramírez-Flandes \& Ulloa, 2008). La secuencia del gen ribosomal 16S fue alineada con las secuencias disponibles en la base de datos Genebank (http://www.ncbi. nlm.nih.gov/genbank/) mediante el software MUSCLE 3,6 (Edgar, 2004). El árbol filogenético de concenso se obtuvo mediante el método de máxima verosimilitud (maximum likelihood) sobre la base del modelo HKY85 (Hasegawa et al., 1985), utilizando inferencia filogenética basada en PhyML (Guindon \& Gascuel, 2003). Adicionalmente, se realizó un análisis de inferencia filogenética mediante el algoritmo de agrupamiento de vecino (neighbor-joining method) utilizando el programa Phylip NJ (Felsenstein, 2005). La evaluación estadística de las topologías de los árboles se realizó por medio de un análisis de bootstrap con 100 repeticiones de muestreo.

ObTención de MOlÉCulas DeSde El SOBRENADANTE LiBRE DE CÉLULAS

La cepa fue incubada durante dos semanas a $20^{\circ} \mathrm{C}$ en medio mínimo R2A, conteniendo $0,15 \%$ de agar. Las células fueron separadas del cultivo por medio de centrifugación a $8.500 \mathrm{rpm}$ durante 20 minutos a $4^{\circ} \mathrm{C}$. Posteriormente, el sobrenadante fue filtrado con un filtro con un tamaño de poro de $0,22 \mu \mathrm{m}$ y liofilizado hasta eliminar todo el medio en un liofilizador Vir Tis 2K (SP Industries, USA). El extracto liofilizado, fue resuspendido en tampón fosfato salino (PBS) obteniendo una solución concentrada 40 veces (40X) en relación a la concentración presente en el cultivo original.

HIDROLISIS DE FICOCOLOIDES

La capacidad hidrolítica del sobrenadante del cultivo se midió mediante el método de Miller (Miller, 1959). Este método consiste en medir los azúcares reductores producidos como resultado de la hidrólisis de agarosa y alginato. Como substrato se utilizaron soluciones de agarosa o alginato al $1 \%(\mathrm{p} / \mathrm{v})$. La reacción se realizó con tres réplicas 
independientes agregando $50 \mu \mathrm{l}$ del extracto concentrado $40 \mathrm{X}$ a $500 \mu \mathrm{l}$ de substrato. La incubación se realizó en un baño termorregulable de agua a tres temperaturas $(4,10 \mathrm{y}$ $30{ }^{\circ} \mathrm{C}$ ) durante $30,60,120,180$ y $240 \mathrm{~min}$. Los valores de concentración de azúcares reductores, -expresada como mg de galactosa por ml- fueron estimados a partir de una curva estándar realizada con galactosa.

\section{ANÁLISIS ESTADÍSTICOS}

La actividad liasa (la capacidad de producir azúcares reductores a partir de diferentes sustratos) fue estimada indirectamente a través de la producción azúcares reductores en dos distintos sustratos. Para evaluar el efecto de la temperatura $\left(4,10\right.$ y $\left.30{ }^{\circ} \mathrm{C}\right)$ sobre la actividad liasa (medida como mg de galactosa por $\mathrm{ml}$ ) con diferentes sustratos (agarosa y alginato) se compararon las pendientes de las rectas de regresión lineal mediante un ANOVA de una vía, utilizando la prueba de Bonferroni para evaluar diferencias a posteriori. Los análisis de regresión lineal y el ANOVA de una vía fueron realizados mediante el programa estadístico
GraphPad Prism versión 4.0 para windows, GraphPad Software, San Diego California USA, www.graphpazd.com.

\section{RESULTADOS}

\section{ASIGNACIÓN TAXONÓMICA}

La comparación de la secuencia obtenida (1448 pb) con las secuencias disponibles en genebank, arrojó alta similitud con secuencias del género Bacillus, en específico con una cepa de Bacillus cereus JN700144.1 (2625 Max Score; 2625 Total Score; $99 \%$ de identidad máxima). El análisis filogenético de 72 secuencias del gen ribosomal 16S provenientes del género Bacillus, obtenido con los métodos de máxima verosimilitud y distancia (neighbor joining) (usando 1086 posiciones de alineamiento; datos no mostrados), indica que la cepa INACH001 forma un clado con Bacillus mycoides (JX144698, JX144699 y JX144726) y Bacillus cereus (JX114949, JX144509, JX144717, JX144723, JX144724 y JX448382) con altos valores de soporte de bootstrap

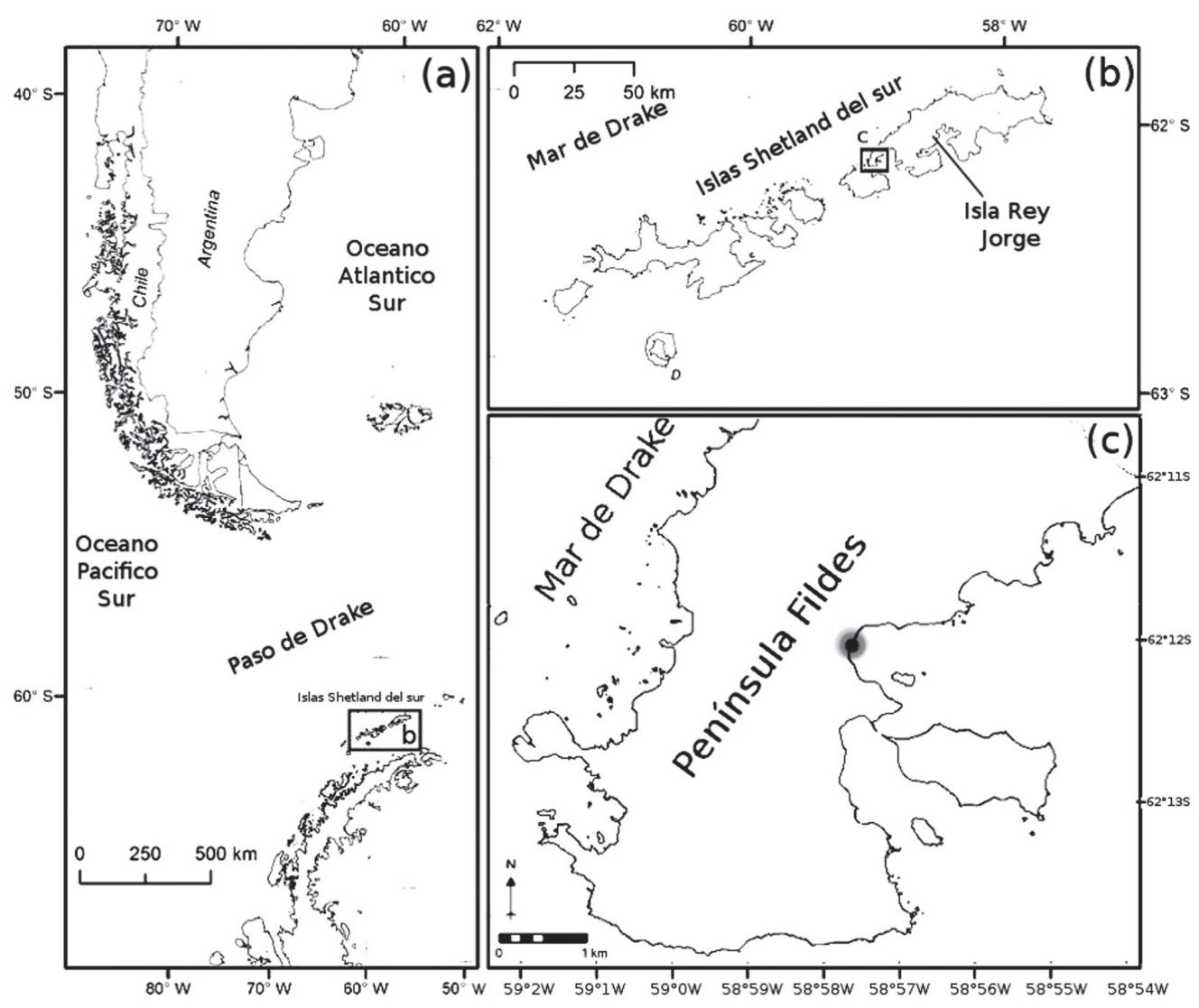

Figura 1. Mapa mostrando la localización de la Península Antártica (a), las islas Shetland del Sur (b) y la Isla Rey Jorge (c). El círculo negro indica el sitio de muestreo ( $\left.\mathrm{S} 62^{\circ} 12^{\prime}, \mathrm{O} 58^{\circ} 57^{\prime}\right)$.

FIgURE 1. Map showing the location of the Antarctic Peninsula (a), South Shetland Islands (b), and King George Island (c). The filled circle indicates the sampling site (S $62^{\circ} 12^{\prime}$, W $\left.58^{\circ} 57^{\prime}\right)$. 


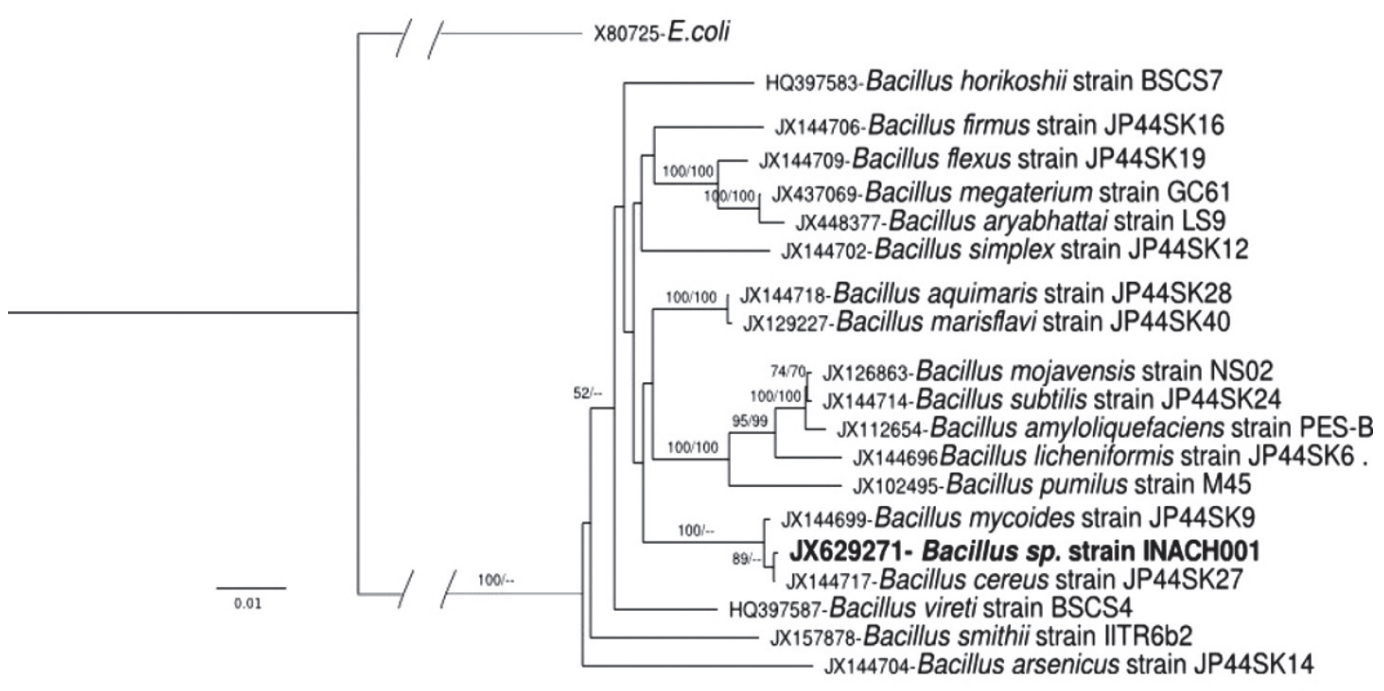

Figura 2: Árbol filogenético de secuencias del gen ribosomal 16S inferido por los métodos de máxima verosimilitud y distancia (usando 1396 posiciones de alineamiento). Los números presentados corresponden a los valores de soporte (valores de bootstrap) de PhyML y Phylip NJ respectivamente. Secuencias utilizadas son presentadas con número de acceso en GenBank seguidas del nombre de la cepa. La secuencia reportada en este trabajo se presenta en negrita.

FIGURE 2: Phylogenetic tree of 16S ribosomal gene sequences inferred from maximum likelihood and distance methods (using alignment of 1396 positions). Numbers presented are support values (bootstrap values) of PhyML and Phylip NJ respectively. Sequences used are presented with GenBank accession number followed by the name of the strain. The sequence reported in this paper is showed in Bold.

(100\%) para ambos métodos. Dentro de este clado la cepa INACH001 se agrupa con las secuencias de Bacillus cereus formando un clado con porcentajes de bootstrap igual a $84 \%$ para el método de máxima verosimilitud y $85 \%$ para el método de distancia (neighbor joining). Una topología similar fue obtenida en el árbol generado con las secuencias de los representantes escogidas al azar (Fig. 2). En conjunto, estos resultados indican que es altamente probable que la cepa INACH-001 pertenezca a Bacillus cereus.

\section{Producción DE AZÚCARES REDUCTORES UTILIZANDO AGAROSA Y ALGINATO COMO SUBSTRATO}

En la fase inicial del experimento (30 primeros minutos), la producción de azúcares reductores de los extractos incubados a temperaturas de $10{ }^{\circ} \mathrm{C}$ y $30{ }^{\circ} \mathrm{C}$ fue cercana a $\sim 0,3 \mathrm{mg}$ de galactosa $/ \mathrm{ml}$, en el medio con agarosa. Esta producción de azúncares fue un $60 \%$ mayor que en el extracto sometido a $4{ }^{\circ} \mathrm{C}$ que presentó una producción de $0,18 \mathrm{mg}$ de galactosa $/ \mathrm{ml}$. No obstante, la producción final de azúcares reductores fue mayor en el extracto sometido a $4{ }^{\circ} \mathrm{C}$ alcanzando un máximo de $0,41 \mathrm{mg}$ de galactosa $/ \mathrm{ml}$. A pesar de lo anterior, el tratamiento sometido a 4 ${ }^{\circ} \mathrm{C}$ no alcanzó su punto de saturación después de cuatro horas (datos no mostrados). Las pendientes de las curvas de producción de galactosa a distintas temperaturas fueron significativamente diferentes $\left(F_{2,11}=9,14 ; P=0,0068\right)$, siendo esta mayor en el extracto sometido a $4{ }^{\circ} \mathrm{C}$ (Fig. $3 \mathrm{a}$ y $3 b)$.

Cuando se usó alginato como sustrato, la producción final de azúcares reductores alcanzó su máximo en el tratamiento de $30{ }^{\circ} \mathrm{C}(1,7 \mathrm{mg}$ de Galactosa $/ \mathrm{ml})$, es decir, 2.3 veces más que lo obtenido a 4 y $10^{\circ} \mathrm{C}$ (Fig. 3c y $3 \mathrm{~d}$ ). Esta producción fue 4,2 veces más que lo obtenido a $4{ }^{\circ} \mathrm{C}$ utilizando agarosa como sustrato. Las pendientes de las curvas de producción galactosa a distintas temperaturas fueron significativamente diferentes $\left(F_{2,11}=95,30 ; P<0,0001\right)$ siendo esta mayor en el extracto sometido a $30^{\circ} \mathrm{C}$ (Fig. $3 \mathrm{c}$ y $\left.3 \mathrm{~d}\right)$.

\section{DISCUSIÓN}

La cepa descrita en este trabajo, perteneciente al género Bacillus (Fig. 2), es capaz de producir azúcares reductores a partir de agarosa a $4{ }^{\circ} \mathrm{C}$ (Fig. 3a). Esta capacidad de degradar agarosa a azúcares más simples ha sido previamente descrita para diversas especies del género Bacillus (e.g., Bacillus megaterium, Bacillus cereus y Bacillus subtilis) aisladas desde sedimento o algas rojas de zonas tropicales (Kim et al., 1999; Khambhaty et al., 2008; Saraswathi 
et al., 2011). No obstante, ninguna de ellas presenta un máximo de actividad a la temperatura encontrada para la cepa de Bacillus reportada en este estudio. Adicionalmente, es importante mencionar que en ninguna cepa de Bacillus, (sean estas provenientes de la Antártica u otros continentes) descritas anteriormente se ha reportado la capacidad conjunta de degradar agar y alginato.

En la mayoría de los estudios, la actividad agarasa se observada cuando los microorganismos son crecidos en un medio con baja concentración de nutrientes y agar como fuente mayoritaria de carbono, fenómeno observado también en la cepa INACH-0001 y que sustenta la hipótesis que plantea que las enzimas agarolíticas, en todos los microorganismos estudiados, están bajo el control de un regulador común
(Shin et al., 2010; Yun et al., 2011). Un fenómeno similar ocurre con la expresión de la alginatoliasa, la cual puede ser inducida cuando son cultivadas en presencia de alginato, como en Alginovibrio aquatilis (Stevens \& Levin, 1977) y otras bacterias marinas como algunas asociadas con Sargassum (Doubet \& Quatrano, 1984; Romeo \& Preston, 1986; Brown \& Preston, 1991). Sin embargo, en algunas especies, como por ejemplo Pseudomonas alginovora, la producción de alginatoliasa es constitutiva (Boyen et al., 1990). Aun cuando la cepa aislada fue crecida en un medio donde la única fuente de carbono correspondía a agar, el sobrenadante libre de células fue capaz de hidrolizar alginato, sugiriendo una posible expresión constitutiva de enzimas degradadoras de otros ficocoloides. (a)

Agarosa

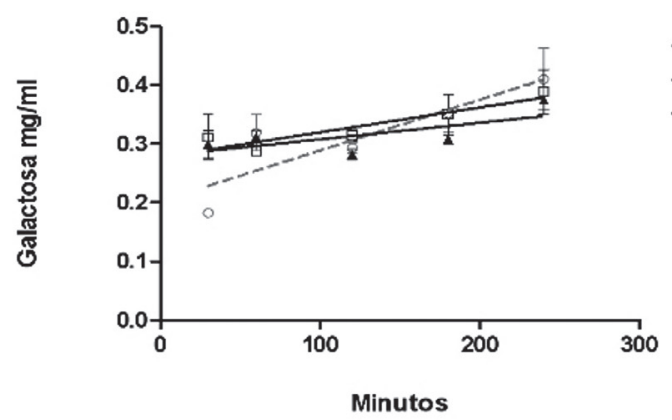

(c)

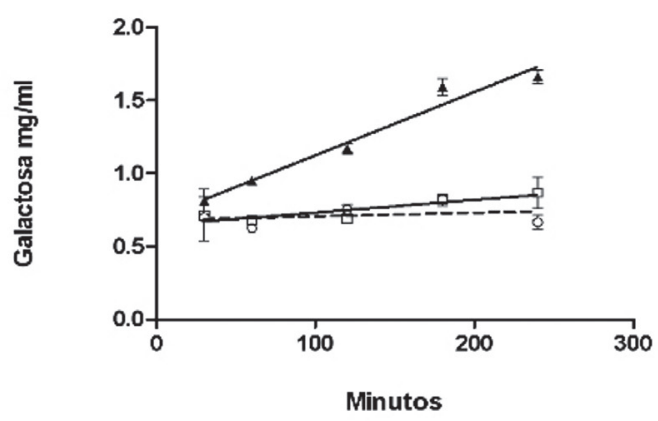

(b)
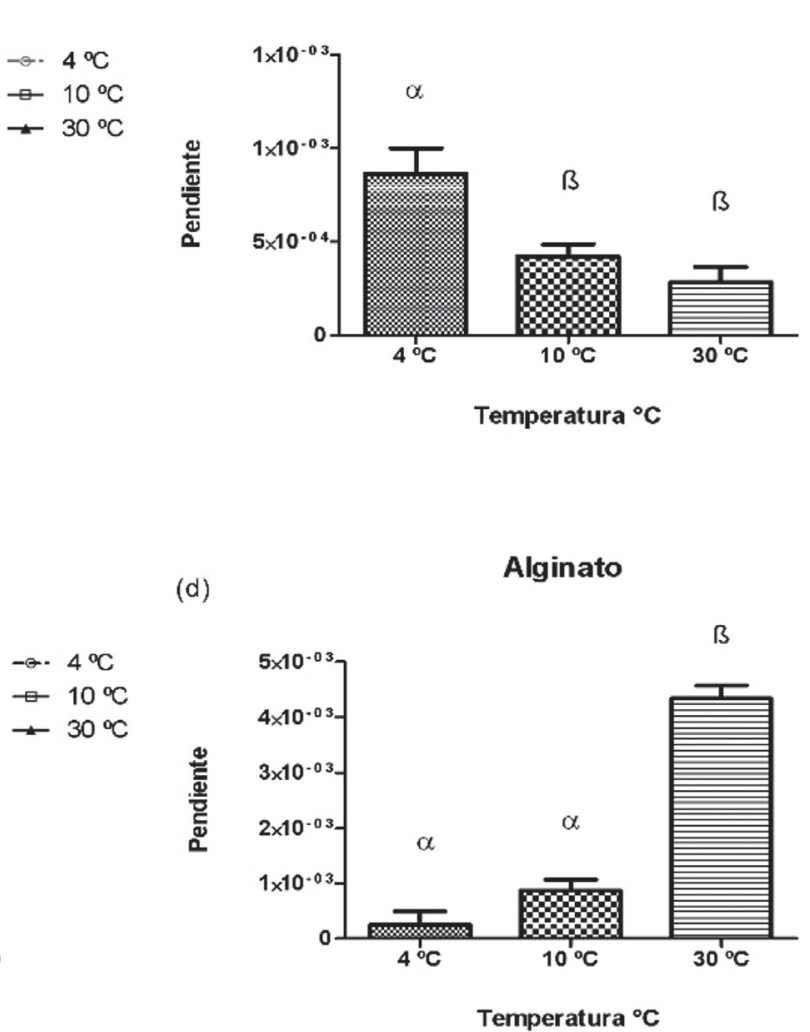

(d)

Alginato

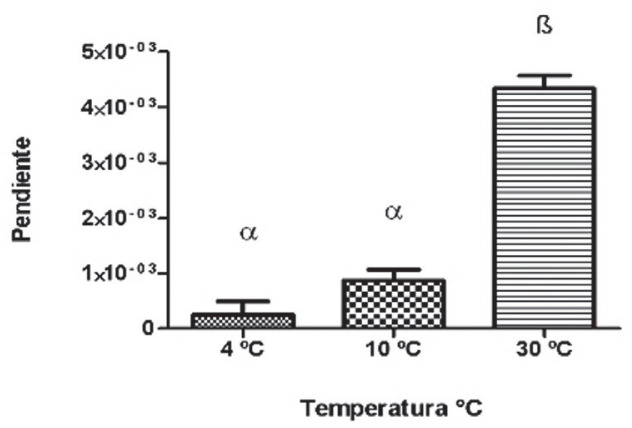

Figura 3 : Producción de azúcares reductoras de extractos de Bacillus sp. (medida como producción de Galactosa) a través del tiempo a tres diferentes temperaturas $\left(4,10\right.$ y $\left.30^{\circ} \mathrm{C}\right)$ en dos diferentes substratos: Agarosa (3a) y Alginato (3c). Efecto de la temperatura (4, 10 y $30{ }^{\circ} \mathrm{C}$ ) en la pendiente de producción de Galactosa a través del tiempo en dos diferentes substratos: Agarosa (3b) y Alginato (3d). Los valores mostrados corresponden a las medias $\pm 1 \mathrm{DE}$ ( $3 \mathrm{a}$ y $3 \mathrm{c}$ ) y $\pm 2 \mathrm{DE}$ (3b y $3 \mathrm{~d}$ ). Las diferentes letras griegas $(\mu, \beta)$ indican diferencias significativas $(P<0.05)$.

FIGURE 3: Reducing sugar production of Bacillus sp.(measured as Galactosa production) through time across three temperatures (4, 10 and $30^{\circ} \mathrm{C}$ ) in two different substrates: Agarose (3a) and Alginate $(3 \mathrm{c})$. Effect of temperature $\left(4,10\right.$, and $\left.30{ }^{\circ} \mathrm{C}\right)$ on the slope of Galactose production through time in two different substrates: Agarose (3b) and Alginate (3d). The values are averages \pm 1 SD $(3 \mathrm{a}$ and $3 \mathrm{c})$ and \pm 1 SD (3b and 3d). Different Greek letters $(\mu, \beta)$ indicate significant differences $(P<0.05)$. 
Las enzimas de microorganismos marinos, especialmente aquellas obtenidas de microorganismos de ambientes extremos, se han hecho cada vez más importantes por el potencial de sus aplicaciones. Es así como, la capacidad de degradar azúcares complejos mediante enzimas específicas se ha reportado como una característica presente en algunas bacterias epífitas de algas (Aoki et al., 1990; Leon et al., 1992; Hosoda et al., 2003; Fu \& Kim, 2010; Kam et al., 2011; Li et al., 2011). En la literatura se pueden encontrar muchos ejemplos como la bacteria marina Saccharophagfus degradans que fue aislada desde Spartina alterniflora en descomposición encontrada en zonas estuarinas (Ekborg et al., 2005). Este microorganismo tiene la capacidad de descomponer el material vegetal completamente debido a un sistema multienzimático que le permite degradar al menos 10 diferentes polisacáridos, entre ellos agar, a sus azúcares elementales o derivados (Shin et al., 2009). La mayoría de las enzimas alginatoliasa aisladas desde microorganismos presentan su actividad máxima a temperaturas que van desde $25^{\circ} \mathrm{C}$ a $50^{\circ} \mathrm{C}$ (Wong et al., 2000).Por otro lado, la mayor parte de las enzimas aisladas y caracterizadas como agarolíticas, tienen un máximo de actividad a temperaturas superiores a $30^{\circ} \mathrm{C}$ (Aoki et al., 1990; Leon et al., 1992; Hosoda et al., 2003; Fu \& Kim, 2010; Kam et al., 2011; Li et al., 2011). Pseudoalteromonas antarctica, una especie marina con actividad agarolítica, fue descrita por Vera et al. (1998) para algas en descomposición presentes en las costas de Valdivia. Estos autores encontraron que las colonias de esta especie tienen una alta capacidad agarolítica, siendo capaces de degradar agar $48 \mathrm{~h}$ después de comenzado el cultivo. La capacidad agarolítica de $P$. antarctica resultó ser mayor a la encontrada por Hosoda et al. (2003) en Paenibacillus spp. la cual comienza a degradar agar después de 10 días comenzado el cultivo. En este caso, ambas bacterias presentan la máxima actividad agarolítica a temperaturas que superan $\operatorname{los} 20^{\circ} \mathrm{C}$.

Considerando el origen antártico de la cepa INACH-0001 $\mathrm{y}$ de poseer un crecimiento entre 4 y $30{ }^{\circ} \mathrm{C}$ (datos no mostrados), estudios futuros a partir de las enzimas aisladas debiesen orientarse a elucidar la diferencia observada en la capacidad de degradar varios substratos a distintas temperaturas. Esta información, además de poseer valor biotecnológico, podría ser extrapolada en un contexto ecológico permitiendo un mayor entendimiento de los procesos de reciclaje del carbono de la biomasa algal varada en la Antártica.

\section{AGRADECIMIENTOS}

Proyecto Innova, Antártica: Fuente de recursos biológicos para la biotecnologia nacional, 07CN13PXT-264; Proyecto Innova Corfo 11IDL2-10651; Fundación Biociencia,
Instituto Antártico Chileno (INACH) por el apoyo logistico. Paris Lavin es financiado por el Proyecto de inserción de capital humano Conicyt 78111301.

\section{REFERENCIAS}

Alkawash, M.A., Soothill, J.S. \& Schiller, N.L. 2006. Alginate lyase enhances antibiotic killing of mucoid Pseudomonas aeruginosa in biofilms. Acta Pathologica, Microbiologica et Immunologica 114: 131-138.

Aoki, T., Araki, T. \& Kitamikado, M. 1990. Purification and characterization of a novel $\beta$-agarase from Vibrio sp. AP-2. European Journal of Biochemistry 187: 461-465.

Athukorala, Y., Jung, W., Vasanthan, T. \& Jeon, Y. 2006. An anticoagulative polysaccharide from an enzymatic hydrolysate of Ecklonia cava. Carbohydrate Polymers 66: 184-191.

AwAD, N. E. 2000. Biologically active steroid from the green alga Ulva lactuca. Phytotherapy Research 14: 641-643.

Boyen C., Bertheau Y., Barbeyron T. \& Kloareg B. 1990. Preparation of guluronate lyase from Pseudomonas alginovora for protoplast isolation in Laminaria. Enzyme and Microbial Technology 12: 885-90

Brown B.J. \& Preston JFIII. 1991. L-Guluronan-specific alginate lyase from a marine bacterium associated with Sargassum. Carbohydrate Research 211: 91-102

Bull, A.T., Ward, A.C. \& Goodfellow, M. 2000. Search and discovery strategies for biotechnology: The paradigm shift. Microbiology and Molecular Biology Reviews 64: 573-606.

Dias, P., Siquieria, J., Vendrusco L., Neiva T., Gagliaerdi A., Maraschi M. \& Ribeiro-do-Valle, R. 2005. Antiangiogenic and antitumoral properties of from the seaweed Sargassum stenophyllum. Cancer Chemotherapy and Pharmacology 56: 436-446.

Donia, M. \& HAMAnN, M.T. 2003 Marine natural products and their potential applications as anti-infective agents, The Lancet Infectious Diseases 3: 338-348

Doubet R.S. \& Quatrano R.S. 1984. Properties of alginate lyases from marine bacteria. Applied and Environmental Microbiology 47: 699-703

EDGAR R.C. 2004. MUSCLE: multiple sequence alignment with high accuracy and high throughput. Nucleic Acids Research 32: 1792-1797.

Ekborg, N., Gonzalez, J., Howard, M.; Taylor, E., Hutcheson, S. \& Weiner, R. 2005. Saccharophagus degradans gen nov., sp. nov., a versatile marine degrader of complex polysaccharides. International Journal of Systematic and Evolutionary Microbiology 55: 1545-1549.

Felsenstein, J. 2005. PHYLIP (Phylogeny Inference Package) Version 3.6. Seattle, WA, USA: Department of Genetics, University of Washington. Distributed by the author.

Fu, X. \& KIm, S. 2010. Agarase: Review of Major Sources, Categories, Purification Method, Enzyme Characteristics and Applications. Marine Drugs 8: 200-218.

Fujihara, M. \& Nagumo, T. 1992. The effect of the content of D-mannuronic acid and L-guluronic acid blocks in alginates on antitumor activity. Carbohydrate Research 
224: 343-347.

Gacesa, P. 1988. Alginates. Carbohydrate Polymers 8: 161-182.

GACESA, P. 1992. Enzymic degradation of alginates. International Journal of Biochemistry 24: 545-552.

Georlette, D., Blaise, V., Collins, T., D’Amico., S., Gratia., E., Hoyoux, A., Marx, J-C., Sonan, G., Feller, G. \& GerDAY., C. 2004. Some like it cold: biocatalysis at low temperatures. FEMS Microbiology Reviews 28: 25-42.

Guindon, S. \& Gascuel, O. 2003. A simple, fast, and accurate algorithm to estimate large phylogenies by maximum likelihood. Systematic Biology 52: 696-704.

Hasegawa M., Kishino H. \& Yano T. 1985. Dating of the humanape splitting by a molecular clock of mitochondrial DNA. Journal of Molecular Biology 22: 160-174.

Hosoda, A., Sakai, M. \& Kanazawa, S. 2003. Isolation and Characterization of Agar-degrading Paenibacillus spp. Associated with the Rhizosphere of Spinach. Bioscience Biotechnology and Biochemistry 67: 1048-1055.

Iтoh, H., Noda, H., Amano, H., Zhuaug, C., Mizuo, T. \& IтоH, H. 1993. Antitumor activity and immunological properties of marine algal polysaccharides, especially fucoidan, prepared from Sargassum thumbergii of Phaeophyceae. Anticancer Research 13: 2045-2052.

Kam N., PARK Y.J., LeE E.Y. \& KIM HS. 2011. Molecular identification of a polyM-specific alginate lyase from Pseudomonas sp. strain KS-408 for degradation of glycosidic linkages between two mannuronates or mannuronate and guluronate in alginate. Canadian Journal of Microbiology, 57: 1032-1041

Khambhaty Y., Mody K. \& JHA B. 2008. Purification, characterization and application of a novel extracellular agarase from a marine Bacillus megaterium. Biotechnology and Bioprocess Engineering 13: 584-591

Kim B.Jo., Kim H.Ju., Duck H.a S., Hee Hwang S., Seok Byun D., Ho Lee T. \& Yul Kong JA. 1999. Purification and characterization of b-agarase from marine bacterium Bacillus cereus ASK202. Biotechnology Letters 21: 10111015

KIN, S.L. 2006. Discovery of novel metabolites from marine actinomycetes. Current Opinion in Microbiology 9: 245251.

Kwon M. \& NAM T. 2007. A polysaccharide of the marine alga Capsosiphon fulvescens induces apoptosis in AGS gasytric cancer cells via an IGF-IR-mediated P13K/Akt pathway. Cell Biology Internacional 31: 765-775.

Leon, O., Quintana, L., Peruzzo, G. \& Slebe, J.C. 1992. Purification and Properties of an Extracellular Agarase from Alteromonas sp. Strain C-1. Applied and Environmental Microbiology 58: 4060-4063.

Li J.W., Dong S., Song J., Li C.B., Chen X.L., Xie B.B. \& Zhang Y.Z. 2011. Purification and Characterization of a Bifunctional Alginate Lyase from Pseudoalteromonas sp. SM0524 Marine Drugs. 9: 109-123.

Matsuhiro B., Conte A.F., Damonte E.B., Kolender A.A., Matulewicz MC., Mejías E.G., Pujol C.A. \& ZúñIga EA. 2005. Structural analysis and antiviral activity of a sulfated galactan from the red seaweed Schizymenia binderi (Gigartinales, Rhodophyta). Carbohydrate Research 15: 2392-2402

Michael, G., Nyval-Collen, P., Barbeyron, T., CzJzek, M.
\& Helbert, W. 2006. Bioconversion of red seaweed galactans: a focus on bacterial agarases and carrageenases. Applied Microbiology and Biotechnology 71: 23-33.

Miller, G.L. 1959. Use of dinitrosalicylic acid reagent for determination of reducing sugar. Analytical Chemistry 31: 426-428.

Nichols, D.S., Sanderson, K., Buia, A.D., van de Kamp, J.L., Holloway, P.E., Bowman, J.P., Smith, M., MancusoNichols, C.A., Nichols, P.D. \& McMeekin, T.A. 2002. Bioprospecting and biotechnology in Antarctica, Conference Proceedings - The Antarctic: Past, Present and Future, 22 June 2001, University of Tasmania, Hobart, Tasmania, pp. 85-105. ISBN $187579624 \mathrm{X}$

Parro, V. \& Mellado, R.P. 1994. Effect of glucose on agarase overproduction in Streptomyces. Gene 145: 49-55.

Ramírez-Flandes, S. \& Ulloa, O. 2008. Bosque: integrated phylogenetic analysis software. Bioinformatics 24: 25392541.

Rasmussen, R.S. \& Morrissey, M.T. 2007. Marine biotechnology for production of food ingredients. Advances In Food And Nutrition Research 52: 237-292

Romeo, T. \& Preston, JFIII. 1986. Purification and structural properties of an extracellular (1-4)- $\beta$-D-mannuronanspecific alginate lyase from a marine bacterium. Biochemistry 25: 8385-8391.

Sarkar, S., Pramanik, A., Mitra A. \& Mukherjee, J. 2010. Bioprocessing data for the production of marine enzymes, Marine Drugs 19: 1323-1327.

Saraswathi, S. Vasanthabharathi, V., Kalaiselvi ,V. \& S. JAYALAKSHMI 2011. Characterization and optimization of agarase from an estuarine Bacillus subtilis. African Journal of Microbiology Research 5: 2960-2968.

Shin, M., Lee, D., Skogerson, K., Wohlgemuth, G., Choi, I.G., FieHn, O. \& Kim, K. 2009. Global metabolic profiling of plant cell wall plysacharide degradation by Saccharophagus degradans. Biotechnology and Bioengineering 105: 477488.

Shin, M., Lee, D., Wohlgemuth, G., Choi, I.G., Fiehn, O. \& Kim, K. 2010. Global metabolite profiling of agarose degradation by Saccharophagus degradans 2-40. New Biotechnology 27: 156-168.

Silva, T., Alves, Queiroz, L., Santos, M., Marques, C., Chavante, S., Rocha, H. \& Leite. 2005. Partial characterization and anticoagulant activity of a heterofucan from the brown seaweed Padina gymnospora. Brazilian Journal of Medical and Biological Research 38: 523-533.

Stach, J.E. 2010. Antimicrobials: treasures from the ocean. Microbiology Today 37: 104-109.

Stevens, R.A. \& Levin RE. 1977. Purification and characteristics of an alginase from Alginovibrio aquatilis. Applied and Environmental Microbiology 33: 1156-1161.

Vera, J., Alvarez, R., Murano, E., Slebe, J.C., \& Leon, O. 1998. Identification of a marine agarolytic Pseudoalteromonas isolate and characterization of its extracellular agarase. Applied and Environmental Microbiology 64: 43784383.

Viana, G. S. B., Freitas, A. L. P., Lima, M. M. L., Vieira, L. A. P., Andrade, M. C. H. \& Benevides, N. M. B. 2002. Antinociceptive activity of sulfated carbohydrates from the red algae Bryothamnion seaforthii (Turner) Kutz. and 
B. triquetrum (SG Gmel) M. Howe. Brazilian Journal of Medical and Biological Research 35: 713-722.

Wong, T.Y., Preston, L.A. \& Schiller, N.L. 2000. Alginate lyase: review of major sources and enzyme characteristics, structure-function analysis, biological roles, and applications. Annual Review of Microbiology 54: 289340.

Xiao, L., Han, F., Yang, Z., Lu, X.Z. \& Yu, W.G. 2006 A novel alginate lyase with high activity on acetylated alginate of Pseudomonas aeruginosa FRD1 from Pseudomonas sp. QD03. World Journal of Microbiology \& Biotechnology 22: 81-88.

Yun E, Shin M, Yoon J-J, Kim Y, Choi I-G \& Kim K. 2011. Production of 3,6-anhydro-l-galactose from agarose by agarolytic enzymes of Saccharophagus degradans 2-40.
Process Biochemistry 46: 88-93.

Zhang C. \& Kim, S.K. 2010. Research and application of marine microbial enzymes: status and prospects. Marine Drugs 8: 1920-1934.

Zhu, W., Chiu, L., Ooi, V., Chan, P. \& Ang, P. 2006. Antiviral property and mechanisms of a sulphated polysaccharide from the brown alga Sargassum patens against herpes simplex virus type 1. Journal of Phytomedicine 13: 695701.

Zoysa, M., Nikapitiya, C., Jeon, Y, Jee, Y. \& Lee, J. 2007. Anticoagulant activity of sulfated polysaccharide isolated from fermented brown seaweed Sargassum fulvellum. Journal of Applied Phycology 20: 67-74.

Recibido: 08.11.12

Aceptado: 14.06.13 